\title{
Soliton-Stripe Patterns in Charged Langmuir Monolayers *†
}

\author{
Xiaofeng Ren \\ Department of Mathematics and Statistics \\ Utah State University \\ Logan, UT 84322-3900, USA
}

\author{
Juncheng Wei \\ Department of Mathematics \\ Chinese University of Hong Kong \\ Shatin, Hong Kong
}

July 16, 2003

\begin{abstract}
We consider a charged Langmuir monolayer problem where electrostatic interaction forces undulations in molecular concentration of the monolayer. Using the $\Gamma$-convergence theory in singular perturbative variational calculus, we prove the existence of soliton-stripe lamellar patterns as one-dimensional local minimizers of the free energy, which are characterized by sharp domain walls delineating fully segregated dense liquid and dilute gas regions of the monolayer.
\end{abstract}

Key words. soliton-stripe pattern, charged Langmuir monolayer, electrostatic interaction, local minimizer, $\Gamma$-convergence

2000 Mathematics Subject Classification. 49J99, 82D99

\section{Introduction}

Amphiphile molecules (surfactants, fatty acids, or lipids) often form insoluble monolayers at the water/air interface. At very low surface pressure such a Langmuir monolayer has small molecular concentration and is in a gaseous phase. An increase in the surface pressure, in some cases, induces an increase of the concentration and consequently a gas to liquid transition. This paper, however, is concerned with an intermediate phase, where the molecular concentration is inhomogeneous with periodic oscillations. In micro-domains where the concentration is small the monolayer is in the gas state, and in micro-domains where the concentration is large the monolayer is in the liquid state. Andelman, Broçhard and Joanny [1] proposed a model for Langmuir monolayers of charged molecules, where the existence of this phase is explained in part by electrostatic interaction.

Let $\phi(r)$ be the relative concentration of the molecules. To eliminate the boundary effect we assume that $r \in \Omega:=(0, L) \times(0, L)$ with the boundaries properly identified so that $\Omega$ becomes topologically a torus. The free energy of the system is

$$
\int_{\Omega}\left(W(\phi)+\frac{b}{2}|\nabla \phi|^{2}\right) d r+\sum_{q \neq(0,0)} \frac{L^{2} e^{2}|\hat{\phi}(q)|^{2}}{2\left(\varepsilon \sqrt{\kappa^{2}+q^{2}}+\varepsilon_{0}|q|\right)} .
$$

${ }^{*}$ Abbreviated title. Soliton-Stripe Patterns.

${ }^{\dagger}$ Corresponding author: Xiaofeng Ren, Phone: (435) 797-0755, Fax: (435) 797-1822, E-mail: ren@math.usu.edu

¥Supported in part by a Direct Grant from CUHK and an Earmarked Grant of RGC of Hong Kong. 
The first part of (1.1)

$$
\int_{\Omega}\left(W(\phi)+\frac{b}{2}|\nabla \phi|^{2}\right) d r
$$

is the standard Ginzburg-Landau free energy. We may take $\left.W(\phi)=(1 / 4)\left((\phi-1 / 2)^{2}-1 / 4\right)\right)^{2}$, whose global minimum value 0 is achieved at $\phi=0$ and $\phi=1$, for simplicity. Then $\phi(r) \approx 0$ means that the monolayer at $r$ is in the gas state and $\phi(r) \approx 1$ the liquid state. This part of the free energy favors segregation of $\phi$ into a large gas region and a large liquid region.

However this phase separation tendency is opposed by the second part of (1.1)

$$
\sum_{q \neq(0,0)} \frac{L^{2} e^{2}|\hat{\phi}(q)|^{2}}{2\left(\varepsilon \sqrt{\kappa^{2}+q^{2}}+\varepsilon_{0}|q|\right)} .
$$

Recall that the layer separates the air and the solution. The charged molecules give an charge distribution $e \phi$ on the monolayer and generate an electric field in the nearby air and the solution. (1.3) is its energy. $\hat{\phi}(q)$ is the Fourier series of $\phi$, i.e.

$$
\hat{\phi}(q)=\frac{1}{L^{2}} \int_{\Omega} \phi(r) e^{-i q \cdot r} d r, \quad \phi(r)=\sum_{q} \hat{\phi}(q) e^{i q \cdot r} .
$$

The wave vector $q$ takes discrete values: $q=\frac{2 \pi n}{L}, n \in \mathbf{Z}^{2}$. $e$ is the electron charge, $\varepsilon_{0}$ the dielectric constant of the air, $\varepsilon$ the dielectric constant of the solution, and $\kappa^{-1}$ the Debye-Hückel screening length. $\varepsilon_{0}$ is smaller than $\varepsilon$. The derivation of this term is found in [1]. It is nonlocal in nature, and its effect is to create undulations in $\phi$.

One important property of the series (1.3) is the $1 /|q|$ decay rate of the coefficients of $|\hat{\phi}(q)|^{2}$. Extensions to more general coefficients may be easily made as long as this decay rate is not exceeded. We choose to work with this particular series for its physical significance.

The soliton-stripe pattern is a particular periodic lamellar pattern of coexisting liquid and gas micro-domains. It is characterized by sharp domain walls (solitons) delineating fully segregated dense liquid and dilute gas regions (stripes), Figure 1 (1). It occurs only at low temperature. The similar phenomenon happens in many other systems including diblock copolymers (Ohta and Kawasaki [17]), Seul-Andelman membranes [30], and smectic films (Selinger et al [29]). In the diblock copolymer theory this pattern is called the strongly segregated lamellar pattern, and in [29] it is called the soliton-stripe pattern. Here we follow the terminology of [29]. We will prove that when (1.2) and (1.3) are properly balanced, i.e. when the parameters in (1.1) are properly chosen, a phase of coexisting liquid and gas micro-domains will appear. The size $L$ of the sample will be determined mathematically. The consequence is that $L$ is several times greater than but still comparable to the size of one micro-domain.

We will show the existence of this pattern using the $\Gamma$-limit theory of De Giorgi [6], which is a rigorous singular perturbation theory in variational calculus. More specifically we will prove that the free energy of the system in one-dimension has local minimizers that have soliton-stripe shape. In the process we will identify the range (see (2.8)) for the parameters $b, e, \varepsilon_{0}, \varepsilon$, and $\kappa$ in (1.1), where the pattern appears. This argument was first used by the authors to study strongly segregated lamellar patterns in di- and tri-block copolymers [19, 20, 24]. We will also determine the optimal thickness of a liquid, or gas, micro-domain.

Compared to the nonlocal interaction term in the diblock copolymer problem, the electrostatic interaction term in (1.1) is more complex. The complexity may be attributed to the slower decay 
(1).

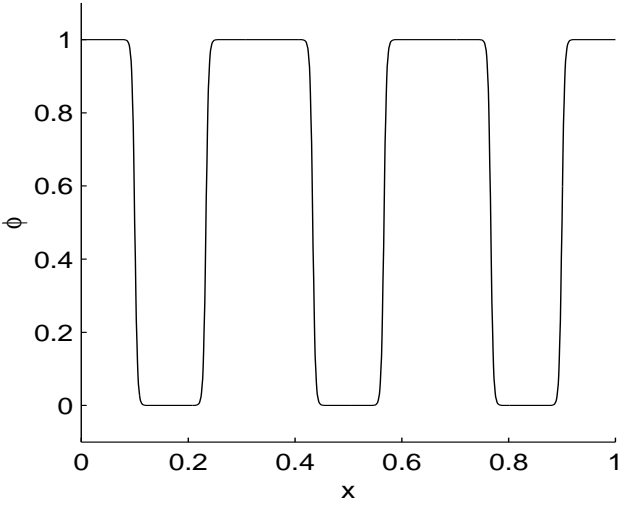

(2).

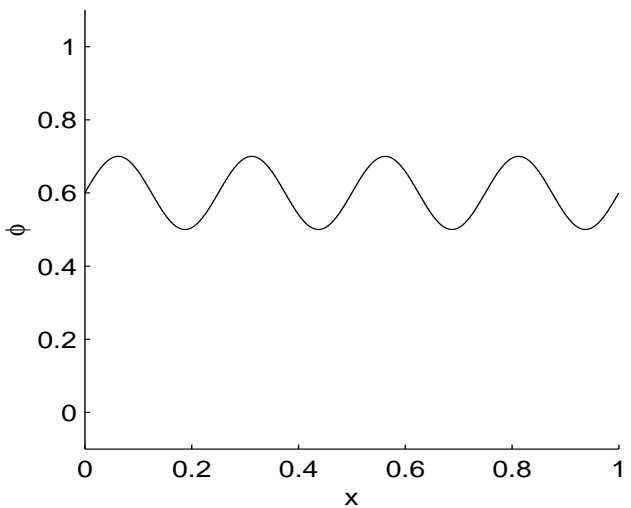

Figure 1: (1). A soliton-stripe pattern for $\phi$ where sharp domain walls separate liquid and gas micro-domains. (2). A sinusoidal pattern which has no sharp domain walls. Gas and liquid are more mixed in (2) than in (1).

rate of the coefficients in (1.3) than that of the diblock copolymer problem, which is $1 /|q|^{2}$ (more on this in Section 5). Our existence result is conditional. For any positive even number $K$ whether there is a locally free energy minimizing, soliton-stripe pattern of $K$ domain walls depends on the positivity of $(K / 2)-1$ numbers: $E(\alpha)$, defined in Theorem 2.1. Only when these numbers are all positive, we have a $K$ wall pattern. However these $E(\alpha)$ 's may be easily numerically calculated, so we are able to give a definitive answer each time the parameters are given. Unlike the diblock copolymer problem, there are cases when some of the $E(\alpha)$ 's are negative and the $\Gamma$-convergence theory does not yield a $K$ wall pattern.

In [1] the electrostatic interaction (1.3) is approximated by a simpler quantity, which they call the dipole-dipole interaction (see (5.9) in Section 5). Our analysis shows that this simplification is unnecessary at least when soliton-stripe patterns are sought. The $\Gamma$-convergence theory can be applied to the original (1.1).

There is another lamellar pattern, in a different parameter range, which has no sharp domain walls. $\phi$ forms a partially segregated, sine-like function in space, Figure 1 (2). This type is termed the weakly segregated lamellar pattern in the diblock copolymer theory, Leibler [12], and the sinusoidal pattern in [29]. It can be treated by the standard bifurcation theory. We sketch this procedure in the last section.

The paper is organized as follows. Section 2 formulates the problem and states the main result, Theorem 2.1. Section 3 explains the $\Gamma$-convergence technique in the construction of local minimizers. The proof of Theorem 2.1 is completed in Section 4. Section 5 includes some remarks.

Mathematical studies on periodic patterns with sharp domain walls started rather recently. Many works have been done to the block copolymer problem. The literature there includes Nishiura and Ohnishi [15], Ohnishi et al [16], Ren and Wei [19, 21, 20, 23, 24, 22, 27], Choksi [3], Fife and Hilhorst [8], Henry [9], and Choksi and Ren [4]. In Ren and Truskinovsky [18] competing oscillation inducing and suppressing interactions are studied in an elastic bar problem. If the nonlocal interaction there is expressed in the Fourier space like here, than the decay rate of coefficients is again of $1 /|q|^{2}$ 
order as in the block copolymer problem. It turns out that the last two problems have much more simpler and explicit nonlocal interactions, so their reduced $\Gamma$-limits are completely solved. The charged monolayer problem studied here has a more implicit nonlocal interaction which is given in the Fourier space. The reduced $\Gamma$-limit is only partially solved. More recently Ren and Wei [25] analyzed the Seul-Andelman membrane problem, where a nonlocal interaction is caused by the bending of the membrane. Finally in [26] a liquid crystal with chirality is investigated. There the nonlocal interaction comes from the director field of the liquid crystal, and because of the unit length constraint on the director field, the nonlocal interaction takes a far more complex, non-quadratic form. Also see Chmaj and Ren [2] where a fully nonlocal model is proposed.

\section{Soliton-stripe pattern}

We scale $\Omega$ to $D=(0,1) \times(0,1)$ to separate the size effect of the sample from its shape effect. Namely we let $\left(x_{1}, x_{2}\right)=\left(r_{1} / L, r_{2} / L\right) \in D$ for $r=\left(r_{1}, r_{2}\right) \in \Omega$. In the Fourier space we introduce $n=\left(\frac{L}{2 \pi}\right) q$. Then (1.1) divided by $L^{2}$ becomes

$$
\int_{D}\left(W(\phi)+\frac{\epsilon^{2}}{2}|\nabla \phi|^{2}\right) d x+\frac{\epsilon}{2} \sum_{n \neq(0,0)} h(n)|\hat{\phi}(n)|^{2} .
$$

We have regarded $\phi$ as a function of the new variable $x$, and $\hat{\phi}$ as a function of $n$. With the new $x$ and $n$

$$
\hat{\phi}(n)=\int_{D} \phi(x) e^{-2 \pi i n \cdot x} d x, \quad \phi(x)=\sum_{n} \hat{\phi}(n) e^{2 \pi i n \cdot x} .
$$

We have introduced a positive parameter $\epsilon$, and defined a function $h$. They are related to the original (1.1) through

$$
\epsilon=\frac{b^{1 / 2}}{L}, \quad h(n)=\frac{L^{2} e^{2}}{2 \pi b^{1 / 2}\left(\varepsilon \sqrt{\left(\frac{\kappa L}{2 \pi}\right)^{2}+n^{2}}+\varepsilon_{0}|n|\right)} .
$$

Since lamellar patterns vary in one direction we assume that $\phi$ depends on $x_{1}$ only, which we denote throughout the rest of the paper by $x$. Also $n$ now becomes an integer. So (2.1) becomes an integral over $[0,1]$ with the periodic boundary condition, not to be confused with the periodic soliton-stripe pattern to be constructed for $\phi$. This means that the points 0 and 1 are identified and we denote this domain by $\mathbf{R} / \mathbf{Z}$. $\mathbf{R} / \mathbf{Z}$ is topologically a circle. On $\mathbf{R} / \mathbf{Z}$ there is the action by the translation group,

$$
\phi(\cdot) \rightarrow \phi(\cdot-y), \forall y \in \mathbf{R} / \mathbf{Z},
$$

so we will use phrases like 'modulo translation' and 'up to translation'. The function $W$ may be generalized from the exact formula mentioned after (1.1). We assume that $W$ is smooth, it has a global minimum value 0 achieved at exactly two points: 0 and 1 , and it grows to $\infty$ at least quadratically fast as its argument approaches $\pm \infty$. We rewrite $(2.1)$ as

$$
I_{\epsilon}(\phi)=\int_{0}^{1}\left(W(\phi)+\frac{\epsilon^{2}}{2} \phi_{x}^{2}\right) d x+\epsilon \sum_{n \in \mathbf{N}} h(n)|\hat{\phi}(n)|^{2},
$$

where

$$
\phi \in W^{1,2}(\mathbf{R} / \mathbf{Z}), \bar{\phi}=m
$$


We have denoted $\bar{\phi}:=\int_{0}^{1} \phi(x) d x$. The constant $m$ is in $(0,1)$. Recall that 0 and 1 are the minima numbers of $W$. The constraint $\bar{\phi}=m$ reflects the fact that the total number of the molecules in the layer is fixed. For technical reasons $I_{\epsilon}$ is trivially extended to $X_{m}$ :

$$
X_{m}=\left\{\phi \in L^{2}(\mathbf{R} / \mathbf{Z}): \bar{\phi}=m\right\}
$$

by taking $I_{\epsilon}(\phi)=\infty$, for $\phi \in X_{m} \backslash W^{1,2}(\mathbf{R} / \mathbf{Z})$.

We will show mathematically that soliton-stripe patterns exist in the parameter range

$$
\epsilon \rightarrow 0 \text {, and } h \text { remains fixed. }
$$

In terms of the original parameters, (2.7) means

$$
b^{1 / 2} \kappa \rightarrow 0, \frac{e^{2}}{\kappa^{2} b^{1 / 2} \varepsilon} \sim 1, \frac{\varepsilon}{\varepsilon_{0}} \sim 1
$$

This parameter range is discovered by the upcoming mathematical argument. The accuracy of the model (1.1) may be tested by comparing (2.8) with that of a real sample with a soliton-stripe pattern. Once (2.8) is satisfied, we take

$$
L \sim \kappa^{-1} .
$$

This $L$ will turn out to be of the same order as the size of a microdomain. Any other order will make the sample either too small or too large, and make mathematical analysis far more complicated. We refer the reader to Müller [14] and [21] where a larger order of $L$ in the diblock copolymer problem is considered, resulting in microdomains having smaller order of size compared to $L$.

The main result of this paper is the following theorem.

Theorem 2.1 Let $h$ be fixed and $K$ a positive even integer. If the following $(K / 2)-1$ numbers $E(\alpha), \alpha=1,2, \ldots(K / 2)-1$,

$$
\begin{aligned}
E(\alpha)= & \frac{K}{4} \sum_{p=1}^{\infty}\left[h\left(\frac{p K}{2}-\left(\frac{K}{2}-\alpha\right)\right)+h\left(\frac{p K}{2}-\alpha\right)-2 h\left(\frac{p K}{2}\right)\right]+\frac{K}{2} \sum_{p=1}^{\infty} h\left(\frac{p K}{2}\right) \cos (2 \pi p m) \\
& -\frac{K}{4}\left|\sum_{p=0}^{\infty} h\left(\frac{p K}{2}+\alpha\right) e^{2 \pi i p m}+\sum_{p=1}^{\infty} h\left(\frac{p K}{2}-\alpha\right) e^{-2 \pi i p m}\right|,
\end{aligned}
$$

are all positive, then $I_{\epsilon}$ has a local minimizer $\phi_{\epsilon}$ of $K$ domain walls when $\epsilon$ is sufficiently small. It has the properties $\lim _{\epsilon \rightarrow 0}\left\|\phi_{\epsilon}-\phi_{0}\right\|_{2}=0$ modulo translation and $\lim _{\epsilon \rightarrow 0} \epsilon^{-1} I_{\epsilon}\left(\phi_{\epsilon}\right)=J\left(\phi_{0}\right)$.

$\|\cdot\|_{2}$ denotes the $L^{2}$-norm. $J$ is defined in (3.4). That $\phi_{\epsilon}$ develops a Soliton-Stripe pattern of $K$ domain walls as $\epsilon \rightarrow 0$ lies in the fact that the limiting profile $\phi_{0}$ of $\phi_{\epsilon}$ is a step function with $K$ 
regularly distributed jump points:

$$
\phi_{0}(x)= \begin{cases}0 & \text { on }\left(0, \frac{1-m}{K}\right), \\ 1 & \text { on }\left(\frac{1-m}{K}, \frac{1+m}{K}\right), \\ 0 & \text { on }\left(\frac{1+m}{K}, \frac{3-m}{K}\right), \\ 1 & \text { on }\left(\frac{3-m}{K}, \frac{3+m}{K}\right), \\ \cdots & \text { on }\left(\frac{K-1-m}{K}, \frac{K-1+m}{K}\right), \\ 0 & \text { on }\left(\frac{K-1+m}{K}, 1\right) .\end{cases}
$$

This theorem reduces the existence of a soliton-stripe pattern of $K$ domain walls to the positivity of $(K / 2)-1$ numbers: $E(\alpha)$. Since these numbers may be accurately calculated numerically, we are able to determine the existence of a soliton-stripe pattern for any given $m, h$, and $K$. Note that when $K$ is 2 , there is no $E(\alpha)$, and the conclusion of the theorem holds true. An interesting case is when $h$ is convex on $[1, \infty)$ and $m=1 / 2$.

Corollary 2.2 If $h$ is convex on $[1, \infty)$ and $m=1 / 2$, then all the $E(\alpha)$ 's are positive and the conclusion of Theorem 2.1 holds.

By (2.3) we see that $h$ is convex on $[1, \infty)$ if $\kappa L$ is small. However we usually want to take the size of the sample $L$ to be sufficiently large, while still in the range (2.9). So for general $h, m$ and $K$, some $E(\alpha)$ may be negative. If this happens, Theorem 2.1 does not yield a soliton-stripe pattern of $K$ domain walls.

Here we run some numerical calculations of $E(\alpha)$. Table 1 shows the smallest $E(\alpha)$, in the second column, for various $K$ when $m=1 / 2$ and $h=\frac{1}{\sqrt{10^{2}+n^{2}}+0.1|n|}$ is non-convex. As indicated in column 3 , there is always a soliton-stripe pattern when $K=2$. But when $K$ is 4 , 6 , or 8 , the $\Gamma$-convergence method employed in this paper does not yield soliton-stripe patterns. The solitonstripe patterns appear when $K \geq 10$. The fourth column and $K_{\text {opt }}$ are explained later. Table 2 shows the calculations for $m=1 / 3$. Again the patterns exist if $K$ is sufficiently large.

Next we address the issue of optimal spacing. We compare the free energy of all the $\phi_{\epsilon}$ 's, whenever they exist, shown in Theorem 2.1, of various numbers of domain walls. The optimal spacing will be detemined by the number of the domain walls of the particular $\phi_{\epsilon}$ that has the least energy. We conjecture that this local minimizer $\phi_{\epsilon}$ is actually the global minimizer of $I_{\epsilon}$. For each $\phi_{\epsilon}$ of $K$ domain walls, we set

$$
\eta(K):=\lim _{\epsilon \rightarrow 0} \epsilon^{-1} I_{\epsilon}\left(\phi_{\epsilon}\right) .
$$

The optimal spacing is defined to be $\frac{L}{K_{o p t}}$ where $K_{o p t}$ is the optimal number of domain walls in the sample that minimizes $\eta$ in positive, even numbers. $\eta(K)$ is given in the next corollary. 


\begin{tabular}{|l|r|r|r|}
\hline$K$ & Smallest $E(\alpha)$ & Existence & $\eta(K)$ \\
\hline \hline 2 & N/A & Yes & $1.5796 \mathrm{e}-02$ \\
\hline 4 & $-3.8851 \mathrm{e}-04$ & No & $1.5115 \mathrm{e}-02$ \\
\hline 6 & $-3.4231 \mathrm{e}-04$ & No & $1.4536 \mathrm{e}-02$ \\
\hline 8 & $-2.0017 \mathrm{e}-04$ & No & $1.4039 \mathrm{e}-02$ \\
\hline 10 & $9.1695 \mathrm{e}-05$ & Yes & $1.3615 \mathrm{e}-02$ \\
\hline 12 & $5.0530 \mathrm{e}-04$ & Yes & $1.3259 \mathrm{e}-02$ \\
\hline 14 & $9.6576 \mathrm{e}-04$ & Yes & $1.2965 \mathrm{e}-02$ \\
\hline 16 & $1.4018 \mathrm{e}-03$ & Yes & $1.2729 \mathrm{e}-02$ \\
\hline 18 & $1.7678 \mathrm{e}-03$ & Yes & $1.2549 \mathrm{e}-02$ \\
\hline 20 & $2.0440 \mathrm{e}-03$ & Yes & $1.2419 \mathrm{e}-02$ \\
\hline 22 & $2.2293 \mathrm{e}-03$ & Yes & $1.2335 \mathrm{e}-02$ \\
\hline 24 & $2.3333 \mathrm{e}-03$ & Yes & $1.2294 \mathrm{e}-02$ \\
\hline $26=K_{\text {opt }}$ & $2.3697 \mathrm{e}-03$ & Yes & $1.2291 \mathrm{e}-02$ \\
\hline 28 & $2.3534 \mathrm{e}-03$ & Yes & $1.2323 \mathrm{e}-02$ \\
\hline 30 & $2.2975 \mathrm{e}-03$ & Yes & $1.2387 \mathrm{e}-02$ \\
\hline
\end{tabular}

Table 1: The existence of soliton-stripe pattern based on our method in the case $m=\frac{1}{2}, h(n)=$ $\frac{1}{\sqrt{10^{2}+n^{2}}+0.1|n|}$, and $\tau=0.0002$.

\begin{tabular}{|l|r|r|r|}
\hline$K$ & Smallest $E(\alpha)$ & Existence & $\eta(K)$ \\
\hline \hline 2 & N/A & Yes & $1.1947 \mathrm{e}-02$ \\
\hline 4 & $-2.8284 \mathrm{e}-04$ & No & $1.1536 \mathrm{e}-02$ \\
\hline 6 & $-2.2176 \mathrm{e}-04$ & No & $1.1202 \mathrm{e}-02$ \\
\hline 8 & $-1.5870 \mathrm{e}-04$ & No & $1.0929 \mathrm{e}-02$ \\
\hline 10 & $-5.3216 \mathrm{e}-05$ & No & $1.0711 \mathrm{e}-02$ \\
\hline 12 & $1.1528 \mathrm{e}-04$ & Yes & $1.0544 \mathrm{e}-02$ \\
\hline 14 & $3.4041 \mathrm{e}-04$ & Yes & $1.0423 \mathrm{e}-02$ \\
\hline 16 & $5.9239 \mathrm{e}-04$ & Yes & $1.0347 \mathrm{e}-02$ \\
\hline $18=K_{\text {opt }}$ & $8.3757 \mathrm{e}-04$ & Yes & $1.0311 \mathrm{e}-02$ \\
\hline 20 & $1.0510 \mathrm{e}-03$ & Yes & $1.0314 \mathrm{e}-02$ \\
\hline 22 & $1.2196 \mathrm{e}-03$ & Yes & $1.0351 \mathrm{e}-02$ \\
\hline 24 & $1.3400 \mathrm{e}-03$ & Yes & $1.0420 \mathrm{e}-02$ \\
\hline 26 & $1.4151 \mathrm{e}-03$ & Yes & $1.0518 \mathrm{e}-02$ \\
\hline 28 & $1.4509 \mathrm{e}-03$ & Yes & $1.0642 \mathrm{e}-02$ \\
\hline 30 & $1.4548 \mathrm{e}-03$ & Yes & $1.0790 \mathrm{e}-02$ \\
\hline
\end{tabular}

Table 2: The existence of soliton-stripe pattern based on our method in the case $m=1 / 3, h(n)=$ $\frac{1}{\sqrt{10^{2}+n^{2}}+0.1|n|}$, and $\tau=0.0002$. 


\section{Corollary 2.3}

$$
\eta(K)=\tau K+\sum_{p=1}^{\infty} \frac{h\left(\frac{p K}{2}\right)(1-\cos (2 \pi p m))}{2 \pi^{2} p^{2}}
$$

In the corollary $\tau$ is a positive constant defined by

$$
\tau=\int_{0}^{1} \sqrt{2 W(u)} d u
$$

It is called the interfacical tension. In the fourth columns of Tables 1 and 2 the values of $\eta(K)$ are calculated. They are minimized at $K_{\text {opt }}$ indicated in the first columns.

For large $K$ we can expand $h(p K / 2)$ and obtain that

$$
\eta(K) \approx \tau K+\frac{1}{K}\left(\frac{L^{2} e^{2}}{2 \pi b^{1 / 2}\left(\varepsilon+\varepsilon_{0}\right)}\right) \sum_{p=1}^{\infty} \frac{1-\cos (2 \pi p m)}{\pi^{2} p^{3}}:=\tau K+\frac{C L^{2}}{K},
$$

where $C$ is a positive constant dependent of $b, e, \varepsilon, \varepsilon_{0}, \kappa$, but independent of $L$. We then find

$$
K_{\text {opt }} \approx\left(\frac{C}{\tau}\right)^{1 / 2} L
$$

The optimal spacing in the original $\Omega$ is then

$$
\frac{2 L}{K_{\text {opt }}} \approx 2\left(\frac{\tau}{C}\right)^{1 / 2}
$$

which is the optimal thickness of a cycle of a liquid region plus a gas region. As it should be, (2.15) is independent of $L$.

Even though the right side of (2.15) is an approximate formula in this context, it is indeed a physically accurate description of optimal spacing, simply because it becomes better approximation as $L$ increases in the range (2.9). It is actually the optimal spacing in the thermodynamic limit.

The next two sections are devoted to the proof of Theorem 2.1 and its corollaries.

\section{$3 \quad$-limit}

The $\Gamma$-limit theory is a singular perturbation theory in the calculus of variations. An introduction to the theory may be found in Dal Maso [5]. In this theory there is a perturbed variational problem, which is often a standard one with a small parameter, say $\epsilon$. The Euler-Lagrange equation of this problem is often a differential equation, although not the case in this paper (5.3). The limiting problem, as $\epsilon \rightarrow 0$, is usually a geometric problem, whose Euler-Lagrange equation is a free boundary problem. Certain properties of the limiting problem are carried over to the perturbed problem (Corollary 3.2). In this sense the perturbed problem is reduced to the limiting problem.

The singular limit (the $\Gamma$-limit) of $\epsilon^{-1} I_{\epsilon}$, denoted by $J$ in this paper, is a variational problem initially defined in

$$
A=\{\phi \in B V(\mathbf{R} / \mathbf{Z},\{0,1\}): \bar{\phi}=m\} .
$$


Here $B V(\mathbf{R} / \mathbf{Z})$ is the class of periodic functions of bounded variation with values in $\{0,1\}$. Each function in $A$ has a finite number of jumps between 0 and 1. A more formal description of these functions may be found in Evans and Gariepy [7, chapter 5]. Naturally for each positive, even integer $K$ we set

$$
A_{K}=\{\phi \in A: \phi \text { has } K \text { jumps }\} \text {. }
$$

Then we have a decomposition

$$
A=\bigcup_{K=2, \text { even }}^{\infty} A_{K}
$$

For each $\phi$ in $A$ we define

$$
J(\phi)=\tau K+\frac{1}{2} \sum_{n \neq(0,0)} h(n)|\hat{\phi}(n)|^{2}, \text { if } \phi \in A_{K} .
$$

Here the positive constant $\tau$ is defined in (2.12). Again we extend $J$ trivially to $X_{m}$ by taking $J(\phi)=\infty$ if $\phi \in X_{m} \backslash A$.

Proposition 3.1 Let $X_{m}$ be equipped with the $L^{2}$ metric.

1. As $\epsilon \rightarrow 0, \epsilon^{-1} I_{\epsilon} \Gamma$-converges to $J$ in the following sense.

(a) For every family $\phi_{\epsilon} \subset X_{m}$ with $\lim _{e \rightarrow 0} \phi_{\epsilon}=\phi$, $\liminf _{\epsilon \rightarrow 0} \epsilon^{-1} I_{\epsilon}\left(\phi_{\epsilon}\right) \geq J(\phi)$;

(b) For every $\phi \in X_{m}$, there is $\left\{\phi_{\epsilon}\right\} \subset X_{m}$ such that $\lim _{\epsilon \rightarrow 0} \phi_{\epsilon}=\phi$ and $\limsup _{\epsilon \rightarrow 0} \epsilon^{-1} I_{\epsilon}\left(\phi_{\epsilon}\right) \leq J(\phi)$.

2. Let $\epsilon_{j}$ be a sequence of positive numbers converging to 0 , and $\left\{\phi_{j}\right\}$ a sequence in $X_{m}$. If $\epsilon_{j}^{-1} I_{\epsilon_{j}}\left(\phi_{j}\right)$ is bounded above in $j$, then $\left\{\phi_{j}\right\}$ is relatively compact in $X_{m}$ and its cluster points belong to $A$.

Proof. We view $\epsilon^{-1} I_{\epsilon}$ as a sum of a local part

$$
K_{\epsilon}(\phi):=\int_{0}^{1}\left[\frac{1}{\epsilon} W(\phi)+\frac{\epsilon}{2} \phi_{x}^{2}\right] d x,
$$

and an $\epsilon$-independent, perturbative, nonlocal part

$$
L(\phi):=\frac{1}{2} \sum_{n \neq 0} h(n)|\hat{\phi}(n)|^{2} .
$$

Regarding $L$, we note that $\phi \rightarrow L(\phi)$ is continuous from $L^{2}(\mathbf{R} / \mathbf{Z})$ to $\mathbf{R}$ by the decay rate of $h$.

After making some minor modifications (change $L^{1}$ to $L^{2}$ ) in the proof of Propositions 1 and 2 of Modica [13], we find that $K_{\epsilon} \Gamma$-converges to $K_{0}$. Here

$$
K_{0}(\phi):=\tau K, \text { if } \phi \in A_{K}
$$

Because $L: X_{m} \rightarrow R$ is a continuous functional, by the definition of $\Gamma$-convergence $\epsilon^{-1} I_{\epsilon}=K_{\epsilon}+L$ $\Gamma$-converges to $J=K_{0}+L$. 
Part 2 of the proposition is a kind of uniform coercivity property. The proof is the same as that of [19, Proposition 2.2].

The next result proved by Kohn and Sternberg [10] asserts that as a corollary of Proposition 3.1 near every isolated local minimizer of $J$ there exists a local minimizer of $I_{\epsilon}$. The original result in [10] deals with a domain with a boundary. Here on $\mathbf{R} / \mathbf{Z}$ we must take care of the translation invariance of $I_{\epsilon}$ and state the result a little differently. Define a manifold of translates of $\phi_{0}$

$$
M\left(\phi_{0}\right):=\left\{\phi \in X_{m}: \phi(\cdot)=\phi_{0}(\cdot-y), y \in \mathbf{R} / \mathbf{Z}\right\}
$$

and a tube like neighborhood of $M\left(\phi_{0}\right)$

$$
N_{\delta}\left(\phi_{0}\right):=\left\{\phi \in X_{m}:\left\|\phi(\cdot)-\phi_{0}(\cdot-y)\right\|<\delta, \text { for some } y \text { in } \mathbf{R} / \mathbf{Z}\right\} .
$$

Corollary 3.2 Let $\delta>0$ and $\phi_{0} \in X_{m}$ be such that $J\left(\phi_{0}\right)<J(\phi)$ for all $\phi \in N_{\delta}\left(\phi_{0}\right) \backslash M\left(\phi_{0}\right)$. Then there exist $\epsilon_{0}>0$ and $\phi_{\epsilon} \in N_{\delta / 2}\left(\phi_{0}\right)$ for all $\epsilon<\epsilon_{0}$ such that $I_{\epsilon}\left(\phi_{\epsilon}\right) \leq I_{\epsilon}(\phi)$ for all $\phi \in N_{\delta / 2}\left(\phi_{0}\right)$. In addition $\phi_{\epsilon} \rightarrow \phi_{0}$ up to translation.

Proposition 3.3 If $\left(x_{1}, x_{2}, \ldots, x_{K}\right)$ strictly minimizes $J$ in $A_{K}$ locally, up to translation, then the corresponding $\phi$ is a strict local minimizer of $J$ in $X_{m}$, modulo translation.

Proof. Suppose that the conclusion is false. There would be a sequence of $\phi_{j}$ such that $\phi_{j} \neq \phi$ modulo translation, $\phi_{j} \rightarrow \phi$ and $J\left(\phi_{j}\right) \leq J(\phi)$. The $L^{2}$-continuity of $L$ implies $\lim _{j \rightarrow \infty} L\left(\phi_{j}\right)=$ $L(\phi)$. Therefore

$$
\limsup _{j \rightarrow \infty} K_{0}\left(\phi_{j}\right) \leq K_{0}(\phi)
$$

On the other hand the lower semicontinuity theorem of BV functions ([7], Theorem 1, p. 172) states

$$
\liminf _{j \rightarrow \infty} K_{0}\left(\phi_{j}\right) \geq K_{0}(\phi)
$$

We deduce that

$$
\lim _{j \rightarrow \infty} K_{0}\left(\phi_{j}\right)=K_{0}(\phi) .
$$

Hence for large $j, \phi_{j}$ has exactly $K$ jumps and is in $A_{K}$. But this is inconsistent with $\phi_{j} \rightarrow \phi$, $J\left(\phi_{j}\right) \leq J(\phi)$, and the assumption of the proposition.

Now the study of $J$ in $X_{m}$ is reduced to the study in $A_{K}$. View the jumps of $\phi: x_{1}, x_{2}, \ldots, x_{K}$ as $K$ points on $(0,1)$, with $0<x_{1}<x_{2}<\ldots<x_{K} \leq 1$, so that

$$
\phi(x)= \begin{cases}0 & \text { on }\left(0, x_{1}\right), \\ 1 & \text { on }\left(x_{1}, x_{2}\right), \\ 0 & \text { on }\left(x_{2}, x_{3}\right), \\ \cdots & \\ 1 & \text { on }\left(x_{K-1}, x_{K}\right), \\ 0 & \text { on }\left(x_{K}, 1\right) .\end{cases}
$$

The constraint $\bar{\phi}=m$ becomes

$$
x_{2}-x_{1}+x_{4}-x_{3}+\ldots+x_{K}-x_{K-1}=m .
$$


The Fourier series of $\phi$ is

$$
\hat{\phi}(n)=\int_{0}^{1} e^{-2 \pi i n x} \phi(x) d x=\frac{1}{2 \pi n} \sum_{j=1}^{K}(-1)^{j} e^{2 \pi i n x_{j}},
$$

and hence

$$
\begin{aligned}
|\hat{\phi}(n)|^{2} & =\frac{1}{4 \pi^{2} n^{2}}\left(\sum_{j=1}^{K}(-1)^{j} e^{2 \pi i n x_{j}}\right)\left(\sum_{k=1}^{K}(-1)^{k} e^{2 \pi i n x_{k}}\right) \\
& =\frac{1}{4 \pi^{2} n^{2}}\left(K+\sum_{j, k ; j \neq k}(-1)^{j+k} \cos \left(2 \pi n\left(x_{j}-x_{k}\right)\right)\right) .
\end{aligned}
$$

$L$ is now viewed as a function of $x_{j}$, and

$$
L\left(x_{1}, x_{2}, \ldots, x_{K}\right)=\sum_{n \in \mathbf{N}} \frac{h(n)}{4 \pi^{2} n^{2}}\left(K+\sum_{j, k ; j \neq k}(-1)^{j+k} \cos \left(2 \pi n\left(x_{j}-x_{k}\right)\right)\right) .
$$

Proposition $3.4 \phi_{0}$ defined in (2.10) or any of its translates is a critical point of $L$ in $A_{K}$.

Proof. The first derivatives of $L$ are

$$
\frac{\partial L}{\partial x_{j}}=-2 \sum_{l \neq j}(-1)^{j+l} \sum_{n \in \mathbf{N}} \frac{h(n)}{2 \pi n} \sin \left(2 \pi n\left(x_{j}-x_{l}\right)\right) .
$$

We evaluate (3.14) at (2.10). Note that there

$$
\begin{aligned}
& \sum_{l \neq j} \sin \left(2 \pi n\left(x_{j}-x_{l}\right)\right) \\
&=\quad \ldots+\sin \left(2 \pi n \frac{4}{K}\right)+\sin \left(2 \pi n \frac{2}{K}\right)+\sin \left(2 \pi n \frac{-2}{K}\right)+\sin \left(2 \pi n \frac{-4}{K}\right)+\ldots \\
& \quad \ldots-\sin \left(2 \pi n\left(x_{j}-x_{j-1}\right)\right)-\sin \left(2 \pi n\left(x_{j}-x_{j+1}\right)\right)-\ldots \\
&= \ldots-\sin \left(2 \pi n\left(x_{j}-x_{j-1}\right)\right)-\sin \left(2 \pi n\left(x_{j}-x_{j+1}\right)\right)- \\
&= \begin{cases}\ldots-\sin \left(2 \pi n \frac{2+2 m}{K}\right)-\sin \left(2 \pi n \frac{2 m}{K}\right)-\sin \left(2 \pi n \frac{-2+2 m}{K}\right)-\ldots & \text { if } j \text { is even } \\
\ldots-\sin \left(2 \pi n \frac{2-2 m}{K}\right)-\sin \left(2 \pi n \frac{-2 m}{K}\right)-\sin \left(2 \pi n \frac{-2-2 m}{K}\right)-\ldots & \text { if } j \text { is odd }\end{cases}
\end{aligned}
$$

Therefore at (2.10)

$$
\frac{\partial L}{\partial x_{1}}=-\frac{\partial L}{\partial x_{2}}=\frac{\partial L}{\partial x_{3}}=\ldots=\frac{\partial L}{\partial x_{K-1}}=-\frac{\partial L}{\partial x_{K}} .
$$

Hence (2.10) is a critical point of $L$ under the constraint (3.10).

\section{Proof of Theorem 2.1}

Because of Corollary 3.2 and Propositions 3.3, we find the spectrum of $L^{\prime \prime}$ at $(2.10)$. The second derivatives of $L$ are

$$
\frac{\partial^{2} L}{\partial x_{j} \partial x_{k}}=2(-1)^{j+k} \sum_{n \in \mathbf{N}} h(n) \cos \left(2 \pi n\left(x_{j}-x_{k}\right)\right) \text { if } j \neq k,
$$




$$
\frac{\partial^{2} L}{\partial x_{j}^{2}}=-2 \sum_{l \neq j}(-1)^{j+l} \sum_{n \in \mathbf{N}} h(n) \cos \left(2 \pi n\left(x_{j}-x_{l}\right)\right) \text { if } j=k .
$$

In this section we translate $(2.10)$ to

$$
y_{0}=0, y_{1}=\frac{1-m}{\nu}, y_{2}=\frac{1}{\nu}, y_{3}=\frac{2-m}{\nu}, y_{4}=\frac{2}{\nu}, \ldots, y_{2 \nu-1}=\frac{\nu-m}{\nu},
$$

where $\nu=K / 2$. For $(4.2)$

$$
\phi_{0}(y)=\left\{\begin{array}{ll}
0 & \text { if } y \in\left(y_{0}, y_{1}\right) \\
1 & \text { if } y \in\left(y_{1}, y_{2}\right) \\
0 & \text { if } y \in\left(y_{2}, y_{3}\right) \\
& \cdots \\
1 & \text { if } y \in\left(y_{2 \nu-1}, 1\right)
\end{array} .\right.
$$

It is more convenient to study the spectrum of $L^{\prime \prime}$ in the complex space $\mathbf{C}^{K}$. We would like to write

$$
\frac{\partial^{2} L}{\partial x_{j}^{2}}=2(-1)^{j+j} \sum_{n \in \mathbf{N}} h(n) \cos \left(2 \pi n\left(y_{j}-y_{j}\right)\right)-2 \sum_{l=0}^{K-1}(-1)^{j+l} \sum_{n \in \mathbf{N}} h(n) \cos \left(2 \pi n\left(y_{j}-y_{l}\right)\right) .
$$

But the two series on the right side are divergent. We have to work with truncated series instead. Denote

$$
\rho_{N}(z)=\sum_{n=1}^{N} h(n) \cos (2 \pi n z) .
$$

Then decompose

$$
\frac{1}{2} L^{\prime \prime}=\lim _{N \rightarrow \infty}\left(\mathbf{E}_{N}+\mathbf{F}_{N}\right)
$$

at (4.2). The $(j, k)$ entry of $\mathbf{E}_{N}$ is $(-1)^{j+k} \rho_{N}\left(y_{j}-y_{k}\right)$. The matrix $\mathbf{F}_{N}$ is a scalar multiple of the identity matrix, i.e.

$$
\mathbf{F}_{N}=\left(-\sum_{l=0}^{K-1}(-1)^{j+l} \rho_{N}\left(y_{j}-y_{l}\right)\right) \mathbf{I}_{K} .
$$

$\mathbf{I}_{K}$ is the $K$ by $K$ identity matrix. The sum in (4.5) is independent of $j$. To see this we note that for each $n$,

$$
\sum_{l=0}^{K-1}(-1)^{j+l} \cos \left(2 \pi n\left(y_{j}-y_{l}\right)\right)=\sum_{\sigma=0}^{\nu-1} \cos \left(2 \pi n \frac{\sigma}{\nu}\right)-\sum_{\sigma=0}^{\nu-1} \cos \left(2 \pi n \frac{\sigma+1-m}{\nu}\right) .
$$

Let us divide $\mathbf{E}_{N}$ into 2 by 2 blocks:

$$
\mathbf{E}_{N}=\left[\begin{array}{rrrr}
\mathbf{e}_{N, 00} & \mathbf{e}_{N, 01} & \ldots & \mathbf{e}_{N, 0(\nu-1)} \\
\mathbf{e}_{N, 10} & \mathbf{e}_{N, 11} & \ldots & \mathbf{e}_{N, 1(\nu-1)} \\
\ldots & & & \\
\mathbf{e}_{N,(\nu-1) 0} & \mathbf{e}_{N,(\nu-1) 1} & \ldots & \mathbf{e}_{N,(\nu-1)(\nu-1)}
\end{array}\right] .
$$


These blocks are labeled by indices $\beta, \xi \in\{0,1, \ldots, \nu-1\}$. A typical $\mathbf{e}_{N, \beta \xi}$ is

$$
\mathbf{e}_{N, \beta \xi}:=\left[\begin{array}{cc}
\rho_{N}\left(y_{2 \beta}-y_{2 \xi}\right) & -\rho_{N}\left(y_{2 \beta}-y_{1+2 \xi}\right) \\
-\rho_{N}\left(y_{1+2 \beta}-y_{2 \xi}\right) & \rho_{N}\left(y_{1+2 \beta}-y_{1+2 \xi}\right)
\end{array}\right] .
$$

The spectral analysis is done in two steps. First we perform a "coarse" discrete Fourier transform, not to be confused with the Fourier transform in (2.4), to convert $\mathbf{E}_{N}+\mathbf{F}_{N}$ to a matrix with vanishing off-diagonal 2 by 2 blocks. In the second step we study the spectra of the diagonal blocks.

The coarse discrete Fourier transform, used in [24] for triblock copolymers, treats a cycle of two micro-domains as a single unit. It is given by the matrix $\mathbf{P}$ whose $(\alpha, \beta)$ block is

$$
\frac{1}{\sqrt{\nu}} \exp \left(-2 \pi i \frac{\alpha \beta}{\nu}\right) \mathbf{I}_{2}, \alpha, \beta \in\{0,1, \ldots, \nu-1\},
$$

where $\mathbf{I}_{2}$ is the 2 by 2 identity matrix. $\mathbf{P}$ is unitary so its inverse $\mathbf{P}^{-1}$ is its adjoint, i.e. (4.8) with the $-2 \pi i$ 's replaced by $2 \pi i$ 's in the exponents. This transform $\mathbf{P}$ is independent of the truncation size $N$. Clearly $\mathbf{P} \mathbf{F}_{N} \mathbf{P}^{-1}=\mathbf{F}_{N}$. The calculation of $\mathbf{P} \mathbf{E}_{N} \mathbf{P}^{-1}$ is a bit more involved. The $(\alpha, \eta)$ block of this product is

$$
\sum_{\beta, \xi} \frac{1}{\nu} \exp \left(-2 \pi i \frac{\alpha \beta}{\nu}+2 \pi i \frac{\xi \eta}{\nu}\right) \mathbf{e}_{N, \beta \xi}
$$

The computation of (4.9) is done on the entries of $\mathbf{e}_{N, \beta \xi}$ individually, so for any $s, t \in\{0,1\}$ the $(s, t)$ entry of $(4.9)$ is

$$
\frac{(-1)^{s+t}}{\nu} \sum_{\beta, \xi} \exp \left(-2 \pi i \frac{\alpha \beta}{\nu}+2 \pi i \frac{\xi \eta}{\nu}\right) \rho_{N}\left(y_{s+2 \beta}-y_{t+2 \xi}\right) .
$$

We define

$$
Q_{N}(\alpha, s, t)= \begin{cases}\sum_{\sigma} \exp \left(-2 \pi i \frac{\alpha \sigma}{\nu}\right) \rho_{N}\left(\frac{\sigma}{\nu}\right) & \text { if } s=t \\ \sum_{\sigma} \exp \left(-2 \pi i \frac{\alpha \sigma}{\nu}\right) \rho_{N}\left(\frac{\sigma}{\nu}-\frac{1-m}{\nu}\right) & \text { if } s=0, t=1 . \\ \sum_{\sigma} \exp \left(-2 \pi i \frac{\alpha \sigma}{\nu}\right) \rho_{N}\left(\frac{\sigma}{\nu}+\frac{1-m}{\nu}\right) & \text { if } s=1, t=0\end{cases}
$$

Then we obtain that

$$
\frac{(-1)^{s+t}}{\sqrt{\nu}} \sum_{\beta} \exp \left(-2 \pi i \frac{\alpha \beta}{\nu}\right) \rho_{N}\left(y_{s+2 \beta}-y_{t+2 \xi}\right)=\frac{(-1)^{s+t}}{\sqrt{\nu}} \exp \left(-2 \pi i \frac{\alpha \xi}{\nu}\right) Q_{N}(\alpha, s, t)
$$

is the $(s, t)$ entry of the $(\alpha, \xi)$ block of $\mathbf{P} \mathbf{E}_{N}$. From (4.9) we conclude that the $(\alpha, \eta)$ block of $\mathbf{P E}_{N} \mathbf{P}^{-1}$ vanishes if $\alpha \neq \eta$ and the $(\alpha, \alpha)$ block is

$$
\left[\begin{array}{rr}
Q_{N}(\alpha, 0,0) & -Q_{N}(\alpha, 0,1) \\
-Q_{N}(\alpha, 1,0) & Q_{N}(\alpha, 1,1)
\end{array}\right]
$$


This way $\mathbf{P}$ diagonalizes $\mathbf{E}_{N}+\mathbf{F}_{N}$ to 2 by 2 blocks for all $N$, where the $\alpha$ 'th diagonal block is

$$
\mathbf{m}_{N, \alpha}=\left[\begin{array}{rr}
Q_{N}(\alpha, 0,0) & -Q_{N}(\alpha, 0,1) \\
-Q_{N}(\alpha, 1,0) & Q_{N}(\alpha, 1,1)
\end{array}\right]-\left(Q_{N}(0,0,0)-Q_{N}(0,0,1)\right) \mathbf{I}_{2} .
$$

Here we have used the fact that

$$
\sum_{k}(-1)^{j+k} \rho_{N}\left(y_{j}-y_{k}\right)=Q_{N}(0,0,0)-Q_{N}(0,0,1)
$$

Now we find more explicit expressions for $Q_{N}(\alpha, s, t)$. To find $Q_{N}(\alpha, 0,0)$ we note

$$
\begin{aligned}
& \sum_{\sigma} \exp \left(-2 \pi i \frac{\alpha \sigma}{\nu}\right) \cos \left(2 \pi \frac{n \sigma}{\nu}\right) \\
& \quad=\frac{1}{2} \sum_{\sigma} \exp \left(-2 \pi i \frac{\alpha \sigma}{\nu}\right)\left(\exp \left(2 \pi i \frac{n \sigma}{\nu}\right)+\exp \left(-2 \pi i \frac{n \sigma}{\nu}\right)\right) \\
& =\frac{1}{2} \sum_{\sigma} \exp \left(2 \pi i \frac{(-\alpha+n) \sigma}{\nu}\right)+\frac{1}{2} \sum_{\sigma} \exp \left(2 \pi i \frac{(-\alpha-n) \sigma}{\nu}\right) \\
& =\left\{\begin{array}{rlr}
0 & \text { if }-\alpha+n \neq 0 \bmod \nu \\
\nu / 2 & \text { if }-\alpha+n \equiv 0 \bmod \nu
\end{array}+\left\{\begin{array}{rr}
0 & \text { if }-\alpha-n \neq 0 \bmod \nu \\
\nu / 2 & \text { if }-\alpha-n \equiv 0 \bmod \nu
\end{array} .\right.\right.
\end{aligned}
$$

Multiplying (4.15) by $h(n)$ and summing over $n$ we deduce

$$
Q_{N}(\alpha, 0,0)=Q_{N}(\alpha, 1,1)=\frac{\nu}{2}\left(\sum_{p=0}^{(N-\alpha) / \nu} h(p \nu+\alpha)+\sum_{p=1}^{(N+\alpha) / \nu} h(p \nu-\alpha)\right) .
$$

Next we compute $Q_{N}(\alpha, 0,1)$. Similar to $(4.15)$ we have

$$
\begin{aligned}
& \sum_{\sigma} \exp \left(-2 \pi i \frac{\alpha \sigma}{\nu}\right) \cos \left(2 \pi \frac{n \sigma-n(1-m)}{\nu}\right) \\
& =\left\{\begin{array}{rl}
0 & \text { if }-\alpha+n \not \equiv 0 \bmod \nu \\
\frac{\nu}{2} e^{-2 \pi i \frac{n(1-m)}{\nu}} & \text { if }-\alpha+n \equiv 0 \bmod \nu
\end{array}+\left\{\begin{array}{rl}
0 & \text { if }-\alpha-n \not \equiv 0 \bmod \nu \\
\frac{\nu}{2} e^{2 \pi i \frac{n(1-m)}{\nu}} & \text { if }-\alpha-n \equiv 0 \bmod \nu
\end{array} .\right.\right.
\end{aligned}
$$

Multiplying the last line by $h(n)$ and summing over $n$ we deduce

$$
Q_{N}(\alpha, 0,1)=\frac{\nu}{2}\left(\sum_{p=0}^{(N-\alpha) / \nu} h(p \nu+\alpha) e^{-2 \pi i \frac{(p \nu+\alpha)(1-m)}{\nu}}+\sum_{p=1}^{(N+\alpha) / \nu} h(p \nu-\alpha) e^{2 \pi i \frac{(p \nu-\alpha)(1-m)}{\nu}}\right) .
$$

The calculations of $Q_{N}(\alpha, 1,0)$ are similar. We find

$$
Q_{N}(\alpha, 1,0)=\frac{\nu}{2}\left(\sum_{p=0}^{(N-\alpha) / \nu} h(p \nu+\alpha) e^{2 \pi i \frac{(p \nu+\alpha)(1-m)}{\nu}}+\sum_{p=1}^{(N+\alpha) / \nu} h(p \nu-\alpha) e^{-2 \pi i \frac{(p \nu-\alpha)(1-m)}{\nu}}\right)
$$


Note that

$$
\begin{aligned}
& Q_{N}(0,0,0)=Q_{N}(0,1,1)=\nu \sum_{p=1}^{N / \nu} h(p \nu), \\
& Q_{N}(0,0,1)=Q_{N}(0,1,0)=\nu \sum_{p=1}^{N / \nu} h(p \nu) \cos (2 \pi p(1-m)),
\end{aligned}
$$

and $Q(\alpha, 0,1)$ is conjugate to $Q(\alpha, 1,0)$. As $N \rightarrow \infty, Q_{N}(\alpha, 0,1)$ and $Q_{N}(\alpha, 1,0)$ are convergent but $Q_{N}(\alpha, 0,0)=Q_{N}(\alpha, 1,1)$ is divergent.

In the second step of our spectral analysis we study $\mathbf{m}_{N, \alpha}$. Note that

$$
\mathbf{m}_{N, 0}=\left[\begin{array}{rr}
Q_{N}(0,0,1) & -Q_{N}(0,0,1) \\
-Q_{N}(0,1,0) & Q_{N}(0,1,0)
\end{array}\right] .
$$

One of the eigenvalues of $\mathbf{m}_{0}$ is 0 and the second is $2 Q_{N}(0,0,1)$. After sending $N \rightarrow \infty$, we have two eigenvalues of $L^{\prime \prime}$ :

$$
0 \text { and } \lim _{N \rightarrow \infty} 2 Q_{N}(0,0,1)=2 \nu \sum_{p=1}^{\infty} h(p \nu) \cos (2 \pi p(1-m)) .
$$

The first eigenvalue comes from the translation invariance of the problem. The second eigenvalue is irrelevant here. Note that an eigenvector of the eigenvalue 0 is $(1,1, \ldots, 1,1)$, in the coordinates before the Fourier transform. The invariant subspace corresponding to $\mathbf{m}_{N, 0}$ is the linear span of the first two columns of $\mathbf{P}$ in (4.8), i.e.

$$
c_{1}(1,0,1,0, \ldots, 1,0)^{T}+c_{2}(0,1,0,1, \ldots, 0,1)^{T} .
$$

In this two-dimensional subspace $(1,-1,1,-1, \ldots, 1,-1)$ is an eigenvector corresponding to the second eigenvalue of $L^{\prime \prime}$. However condition (3.10) requires that any eigenvector $\left(z_{1}, z_{2}, \ldots, z_{K}\right)$ must satisfy

$$
z_{2}-z_{1}+z_{4}-z_{3}+\ldots+z_{K}-z_{K-1}=0 .
$$

The eigenvector $(1,-1,1,-1, \ldots, 1,-1)$ does not satisfy (4.21). It is actually perpendicular to the plane (4.21). Thus the second eigenvalue is excluded.

When $\alpha>0$, the two eigenvalues of $\mathbf{m}_{N, \alpha}$ are $Q_{N}(\alpha, 0,0)+\left|Q_{N}(\alpha, 0,1)\right|-Q_{N}(0,0,0)+Q_{N}(0,0,1)$ and $Q_{N}(\alpha, 0,0)-\left|Q_{N}(\alpha, 0,1)\right|-Q_{N}(0,0,0)+Q_{N}(0,0,1)$. From $(4.16,4.17,4.18,4.19)$ we find them to be

$$
\begin{aligned}
& \frac{\nu}{2}\left(\sum_{p=0}^{(N-\alpha) / \nu} h(p \nu+\alpha)+\sum_{p=1}^{(N+\alpha) / \nu} h(p \nu-\alpha)\right)-\nu \sum_{p=1}^{N / \nu} h(p \nu)+\nu \sum_{p=1}^{N / \nu} h(p \nu) \cos (2 \pi p m) \\
& \pm \frac{\nu}{2}\left|\sum_{p=0}^{(N-\alpha) / \nu} h(p \nu+\alpha) e^{-2 \pi i p m}+\sum_{p=1}^{(N+\alpha) / \nu} h(p \nu-\alpha) e^{2 \pi i p m}\right| .
\end{aligned}
$$

The smaller one in (4.22) takes - in \pm , which, after $N$ is sent to $\infty$, yields $E(\alpha)$ in Theorem 2.1. Note that the first three series must be combined into one before $N$ is sent to $\infty$ for the sake of convergence. This completes the proof of the theorem. 
Proof of Corollary 2.2. When $m=1 / 2$, the $E(\alpha)$ 's are simplified to

$$
\begin{gathered}
\frac{\nu}{2} \sum_{p=1}^{\infty}[h(p \nu-(\nu-\alpha))+h(p \nu-\alpha)-2 h(p \nu)]+\nu \sum_{p=1}^{\infty} h(p \nu)(-1)^{p} \\
-\frac{\nu}{2}\left|-\sum_{p=1}^{\infty} h(p \nu-(\nu-\alpha))(-1)^{p}+\sum_{p=1}^{\infty} h(p \nu-\alpha)(-1)^{p}\right| .
\end{gathered}
$$

When the quantity in $|\ldots|$ is non-negative, (4.23) is

$$
\begin{aligned}
= & \frac{\nu}{2} \sum_{p=1}^{\infty}\left[h(p \nu-(\nu-\alpha))+h(p \nu-\alpha)-2 h(p \nu)+2(-1)^{p} h(p \nu)\right. \\
& \left.+(-1)^{p} h(p \nu-(\nu-\alpha))-(-1)^{p} h(p \nu-\alpha)\right] \\
= & \frac{\nu}{2} \sum_{p=1}^{\infty} \begin{cases}2 h(p \nu-\alpha)-4 h(p \nu) & \text { if } p \text { is odd } \\
2 h(p \nu-(\nu-\alpha)) & \text { if } p \text { is even }\end{cases} \\
= & \nu \sum_{p=1, o d d}^{\infty}[h(p \nu-\alpha)+h(p \nu-(\nu-\alpha))-2 h(p \nu)]>0,
\end{aligned}
$$

where the last inequality follows from the convexity of $h$. When the quantity in $|\ldots|$ is negative, $(4.23)$ is

$$
\begin{aligned}
= & \frac{\nu}{2} \sum_{p=1}^{\infty}\left[h(p \nu-(\nu-\alpha))+h(p \nu-\alpha)-2 h(p \nu)+2(-1)^{p} h(p \nu)\right. \\
& \left.-(-1)^{p} h(p \nu-(\nu-\alpha))+(-1)^{p} h(p \nu-\alpha)\right] \\
= & \frac{\nu}{2} \sum_{p=1}^{\infty} \begin{cases}2 h(p \nu-(\nu-\alpha))-4 h(p \nu) & \text { if } p \text { is odd } \\
2 h(p \nu-\alpha) & \text { if } p \text { is even }\end{cases} \\
= & \nu \sum_{p=1, \text { odd }}^{\infty}[h(p \nu+\nu-\alpha)+h(p \nu-(\nu-\alpha))-2 h(p \nu)]>0,
\end{aligned}
$$

where the last inequality again follows from the convexity of $h$. $\square$

Proof of Corollary 2.3. We find $J$ at every (2.10):

$$
\begin{aligned}
\eta(K) & =\lim _{\epsilon \rightarrow 0} \epsilon^{-1} I_{\epsilon}\left(\phi_{\epsilon}\right)=J\left(\phi_{0}\right) \\
& =\tau K+\sum_{n \in \mathbf{N}} \frac{K h(n)}{4 \pi^{2} n^{2}} \sum_{j=1}^{K}(-1)^{j+k} \cos \left(2 \pi n\left(x_{j}-x_{k}\right)\right) \\
& =\tau K+\sum_{n \in \mathbf{N}} \frac{K h(n)}{4 \pi^{2} n^{2}}\left(\sum_{\sigma=0}^{\nu-1}\left(\cos \left(2 \pi \frac{n \sigma}{\nu}\right)-\cos \left(2 \pi\left(\frac{n \sigma+n(1-m)}{\nu}\right)\right)\right)\right. \\
& =\tau K+\sum_{p=1}^{\infty} \frac{h\left(\frac{p K}{2}\right)(1-\cos (2 \pi p(1-m)))}{2 \pi^{2} p^{2}} \cdot
\end{aligned}
$$




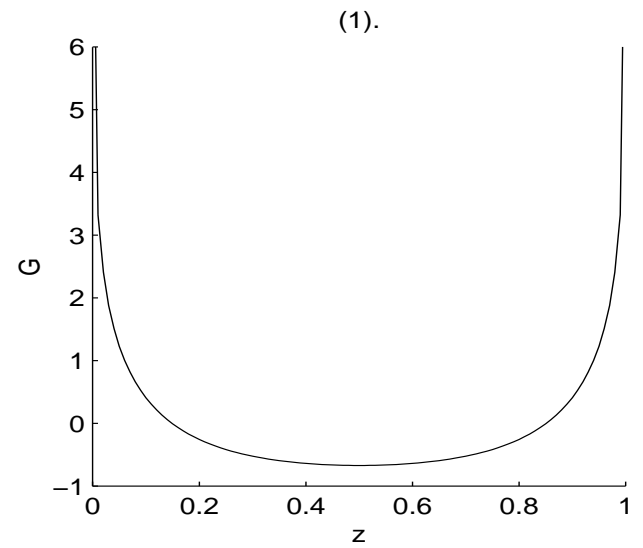

Figure 2: Let $h(n)=\frac{1}{\sqrt{1+n^{2}}+0.5|n|}$. (1). $G$ on $[0,1] .(2)$. $G$ on $[0.5,1.5]$.



\section{Remarks}

Theorem 2.1 shows the existence of soliton-stripe patterns as local minimizers of $I_{\epsilon}$. Many questions remain here and the overall structure of $I_{\epsilon}$ is still obscure. Regarding the reduced problem $J$ we have only showed in Proposition 3.4 that the cyclic $\phi_{0}$ 's are critical points. Here cyclic refers to the fact that the jump points are regularly distributed (2.10). But it is not clear whether $J$ has other non-cyclic critical points. Without knowing all the critical points of $J$ we are unable to identify the global minimizers of $J$ and $I_{\epsilon}$, although we suspect that the global minimizer of $J$ is the $\phi_{0}$ with the optimal spacing.

Although there exist cyclic $\phi_{0}$ 's that are critical points but not local minimizers of $J$, it is not clear to us whether they correspond to soliton-stripe patterns as unstable critical points of $I_{\epsilon}$. This is because that the $\Gamma$-convergence theory only reduces the existence of local minimizers, not unstable critical points, of $I_{\epsilon}$ to those of $J$.

It is natural, as done by the authors in the diblock copolymer problem [22], to contemplate the two-dimensional stability of the one-dimensional local minimizers viewed in two-dimensions. We suspect, based on our experience in [22], that not all the one-dimensional local minimizers constructed in Theorem 2.1 are stable in two-dimensions. The one with $K=2$ and some other ones with smaller $K$ values may be unstable. There is also the possibility, as in the diblock copolymer problem [27], that there could be stable lamellar patterns with wriggled domain walls in two-dimensions.

The free energy (2.4) may also be written as

$$
I_{\epsilon}(\phi)=\int_{0}^{1}\left(W(\phi)+\frac{\epsilon^{2}}{2} \phi_{x}^{2}\right) d x+\frac{\epsilon}{2} \int_{0}^{1} \int_{0}^{1} G(x-y) \phi(x) \phi(y) d x d y,
$$

where

$$
G(z)=\sum_{n \neq 0} h(n) e^{2 \pi i n z}
$$

A numerically calculated $G$ is shown in Figure 2. The local minimizers of $I_{\epsilon}$ constructed in Theorem 
(1).

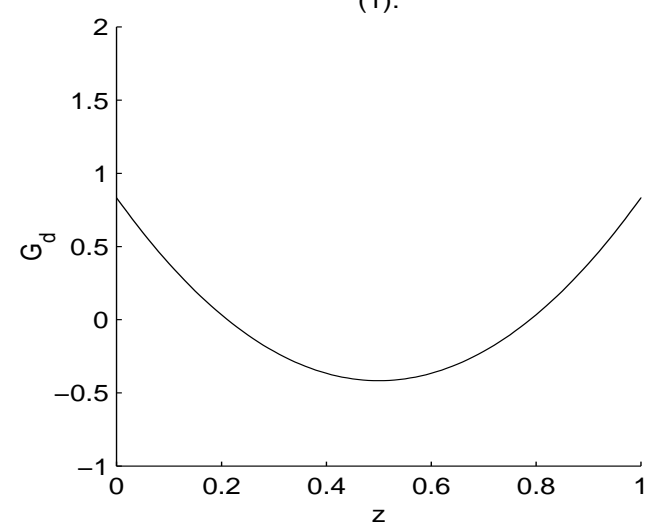

(2).

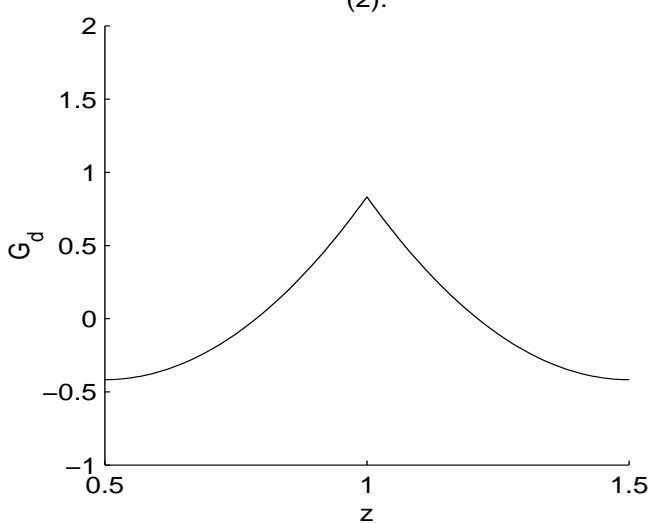

Figure 3: $G_{d}(z)=10\left(\frac{z^{2}}{2}-\frac{z}{2}+\frac{1}{12}\right)$ of the diblock copolymer problem. (1). $G_{d}$ on $[0,1] .(2) . G_{d}$ on $[0.5,1.5]$.

2.1 satisfy the Euler-Lagrange equation

$$
W^{\prime}(\phi)-\epsilon^{2} \phi_{x x}+\epsilon G[\phi]=\text { Const. }
$$

of (5.1). We have introduced the integral operator

$$
G[\phi](x)=\int_{0}^{1} G(x-y) \phi(y) d y .
$$

The Const. on the right side of (5.3) is a Lagrange multiplier coming from the constraint $\bar{\phi}=m$. Integrating (5.3), we find this Const. $=\overline{W^{\prime}(\phi)}$.

The diblock copolymer problem formulated on $\mathbf{R} / \mathbf{Z}$ has a similar expression of free energy. The difference is in the function $G$. In the diblock copolymer problem, one uses

$$
G_{d}(z)=\gamma\left(\frac{z^{2}}{2}-\frac{z}{2}+\frac{1}{12}\right), z \in[0,1] \text {, and periodically extended to } \mathbf{R}
$$

in place of $G$ in (5.1), Figure 3. $\gamma$ in $G_{d}$ is a positive constant. This explicitly given $G_{d}$, without $\gamma$, is the Green function of the operator $-\frac{d^{2}}{d x^{2}}$. In the Fourier space corresponding to $h$ is

$$
h_{d}(n)=\frac{\gamma}{4 \pi^{2} n^{2}} .
$$

Note that $G_{d}$ in the diblock copolymer problem is bounded but $G$ in the charged Langmuir monolayer problem has singularity at $\mathbf{Z}$.

There are subtle consequences following the difference. Firstly in the diblock copolymer problem for each even $K$ there is always a $K$ domain wall soliton-stripe pattern as a one-dimensional local minimizer. The pattern comes from (2.10), which is the only critical point of the corresponding $L$. This fact is established for the diblock copolymer problem under the natural boundary condition in 
[19]. The same fact holds under the periodic boundary condition after we make some small changes in [19]. In the charged Langmuir monolayer problem the existence of a $K$ wall soliton-stripe pattern as a local minimizer is conditioned on the positivity of the $E(\alpha)$ 's, Theorem 2.1.

Secondly following (5.5) or (5.6) one finds different optimal spacing. The dependence of the reduced free energy there on $K$ takes the form

$$
\eta_{d}(K)=\tau K+\frac{C L^{3}}{K^{2}}
$$

for some positive constant $C$ independent of $L$. It leads to

$$
K_{\text {opt }} \approx\left(\frac{2 C}{\tau}\right)^{1 / 3} L
$$

Note the difference of the exponent $1 / 3$ in (5.8) and the exponent $1 / 2$ in (2.14). Formulae (5.7) and (5.8) also appear in the Seul-Andelman membrane problem [25], and the chiral liquid crystal problem [26]. On the other hand (2.14) is found in the domain structures of ferromagnets, Landau, Lifshitz and Pitaevskii [11], and superconductors in the intermediate state, Tinkham [31].

Andelman et al [1] also considered neutral Langmuir monolayers. They postulated a free energy

$$
\int_{\Omega}\left(W(\phi)+\frac{b}{2}|\nabla \phi|^{2}\right) d r+\sum_{q \neq(0,0)} \frac{-L^{2} \mu^{2} \varepsilon_{0}|q \| \hat{\phi}(q)|^{2}}{2 \varepsilon\left(\varepsilon+\varepsilon_{0}\right)} .
$$

This expression may also be formally regarded as an approximation of (1.1) when $|q|$ is small. However if the soliton-stripe pattern is sought, (5.9) can not be handled the way we did (1.1), because the last term of (5.9) is divergent when $\phi$ is a step function. In [1] a cutoff was introduced to deal with the divergence. Without this modification it is doubtful that (5.9) admits soliton-stripe patterns.

The sinusoidal lamellar pattern, Figure 1 (2), mentioned in the introduction is of very different nature. It bifurcates out of the constant solution $m$ of (5.3). To see this we note that the eigenvalue problem of $(5.3)$ at $\phi$ is

$$
W^{\prime \prime}(\phi) \psi-\overline{W^{\prime \prime}(\phi) \psi}-\epsilon^{2} \psi_{x x}+\epsilon G[\psi]=\lambda \psi
$$

Equation (5.3) is satisfied by $\phi=m$. At this $m$, we have, in (5.10),

$$
\psi=\cos (2 n \pi x), \text { or } \psi=\sin (2 n \pi x), n=1,2,3, \ldots
$$

and the corresponding

$$
\lambda=W^{\prime \prime}(m)+4 \epsilon^{2} n^{2} \pi^{2}+\epsilon h(n), n=1,2,3, \ldots
$$

Depending on $\epsilon, h$, and $m$ the principal eigenvalue (i.e. the smallest $\lambda$ ) may be positive, negative, or zero. This allows one to use the bifurcation theory to find solutions bifurcating out of $m$. The phenomenon occurs in a parameter range different from (2.8). Such solutions differ from $m$ by a function proportional to (5.11), to the first order approximation. We then obtain a sinusoidal lamellar pattern. Whether these solutions are stable, an important issue in the physical model, may also be determined by examining the shape of the bifurcation diagram. This construction is rather standard, so we omit the detail. One difficulty here is the multiplicity of the eigenvalues (5.12) due to the translation group action on $\mathbf{R} / \mathbf{Z}$. Hence a group invaraiant version of the bifurcation theory is needed. The reader may find all the necessary tools in Sattinger [28].

Acknowledgments. The support from Institute of Mathematical Sciences at Chinese University of Hong Kong is very much appreciated. We thank one referee for many valuble suggestions. 


\section{References}

[1] D. Andelman, F. Broçhard, and J.-F. Joanny. Phase transitions in Langmuir monolayers of polar molecules. J. Chem. Phys., 86(6):3673-3681, 1987.

[2] A. Chmaj and X. Ren. Multiple layered solutions of the nonlocal bistable equation. Physica D, 147(1-2):135-154, 2000.

[3] R. Choksi. Scaling laws in microphase separation of diblock copolymers. J. Nonlinear Sci., 11:223-236, 2001.

[4] R. Choksi and X. Ren. On the derivation of a density functional theory for microphase separation of diblock copolymers. J. Statist. Phys., 113(1\&2):151-176, 2003.

[5] G. Dal Maso. Introduction to Gamma-Convergence. Progress in Nonlinear Differential Equations and Their Applications, Vol 8. Birkhäuser, Boston, 1992.

[6] E. De Giorgi. Sulla convergenza di alcune successioni di integrali del tipo della'area. Rendiconti di Matematica, 8:277-294, 1975.

[7] L.C. Evans and R.F. Gariepy. Measure Theory and Fine Properties of Functions. CRC Press, Boca Raton, New York, London, Tokyo, 1992.

[8] P.C. Fife and D. Hilhorst. The Nishiura-Ohnishi free boundary problem in the 1D case. SIAM J. Math. Anal., 33(3):589-606, 2001.

[9] M. Henry. Singular limit of a fourth order problem arising in the micro-phase separation of diblock copolymers. Adv. Differential Equations, 6(9):1049-1114, 2001.

[10] R. Kohn and P. Sternberg. Local minimisers and singular perturbations. Proc. Royal Soc. Edin., 111A:69-84, 1989.

[11] L.D. Landau, E.M. Lifshitz, and L.P. Pitaevskii. Electrodynamics of Continuous Media, Course of Theoretical Physics, Volume 8. Butterworth-Heinemann, second edition, 1984.

[12] L. Leibler. Theory of microphase separation in block copolymers. Macromolecules, 13(6):16021617, 1980.

[13] L. Modica. The gradient theory of phase transitions and the minimal interface criterion. Arch. Rat. Mech. Anal., 98:357-383, 1987.

[14] S. Müller. Singular perturbations as a selection criterion for periodic minimizing sequences. Calc. Var. Partial Differential Equations, 1(2):169-204, 1993.

[15] Y. Nishiura and I. Ohnishi. Some mathematical aspects of the microphase separation in diblock copolymers. Physica D, 84:31-39, 1995.

[16] I. Ohnishi, Y. Nishiura, M. Imai, and Y. Matsushita. Analytical solutions describing the phase separation driven by a free energy functional containing a long-range interaction term. Chaos, $9(2): 329-341,1999$. 
[17] T. Ohta and K. Kawasaki. Equilibrium morphology of block copolymer melts. Macromolecules, 19(10):2621-2632, 1986.

[18] X. Ren and L. Truskinovsky. Finite scale microstructures in nonlocal elasticity. In recognition of the sixtieth birthday of Roger L. Fosdick (Blacksburg, VA, 1999). J. Elasticity, 59(1-3):319-355, 2000.

[19] X. Ren and J. Wei. On the multiplicity of solutions of two nonlocal variational problems. SIAM J. Math. Anal., 31(4):909-924, 2000.

[20] X. Ren and J. Wei. Concentrically layered energy equilibria of the di-block copolymer problem. European J. Appl. Math., 13(5):479-496, 2002.

[21] X. Ren and J. Wei. On energy minimizers of the di-block copolymer problem. Interfaces Free Bound., 5:193-238, 2003.

[22] X. Ren and J. Wei. On the spectra of 3-D lamellar solutions of the diblock copolymer problem. SIAM J. Math. Anal., 35(1):1-32, 2003.

[23] X. Ren and J. Wei. Triblock copolymer theory: Free energy, disordered phase and weak segregation. Physica D, 178:103-117, 2003.

[24] X. Ren and J. Wei. Triblock copolymer theory: Ordered ABC lamellar phase. J. Nonlinear Sci., 45(2):175-208, 2003.

[25] X. Ren and J. Wei. The soliton-stripe pattern in the Seul-Andelman membrane. Phsica D, in press.

[26] X. Ren and J. Wei. Molecular chirality and soliton-stripe pattern in liquid crystal films. preprint.

[27] X. Ren and J. Wei. Wriggled lamellar solutions and their stability in the diblock copolymer problem. preprint.

[28] D. Sattinger. Group theoretic methods in bifurcation theory. Springer-Verlag, New York, 1979.

[29] J.V. Selinger, Z.-G. Wang, R.F. Bruinsma, and C.M. Knobler. Chiral symmetry breaking in Langmuir monolayers and smectic films. Phys. Rev. Lett., 70(8):1139-1142, 1993.

[30] M. Seul and D. Andelman. Domain shapes and patterns: The phenomenology of modulated phases. Science, 267:476-483, 1995.

[31] M. Tinkham. Introduction to Superconductivity. McGraw-Hill, second edition, 1995. 